PROCEEDINGS OF THE

AMERICAN MATHEMATICAL SOCIETY

Volume 130, Number 3, Pages 833-839

S 0002-9939(01)06121-4

Article electronically published on June 21, 2001

\title{
CATEGORY BOUNDS FOR NONNEGATIVE RICCI CURVATURE MANIFOLDS WITH INFINITE FUNDAMENTAL GROUP
}

\author{
JOHN OPREA
}

(Communicated by Wolfgang Ziller)

\begin{abstract}
This brief note presents refinements of the bounds on the first Betti number and the polynomial growth degree of the fundamental group for manifolds with nonnegative Ricci curvature and infinite fundamental group. These refinements are then sharpened when applied to symplectic manifolds.
\end{abstract}

\section{INTRODUCTION}

It is by now well-established that the condition of having nonnegative Ricci curvature constrains a Riemannian manifold in many ways. For instance, if the Ricci curvature is nonnegative and bounded away from zero, then Myers' theorem (see $\mathrm{DoC}$ or GHL for example) shows that the manifold is compact with finite fundamental group. The Bochner method (see [LM] or [GHL]) may be applied to a manifold $M$ with nonnegative Ricci curvature to constrain the first Betti number by $b_{1}(M) \leq \operatorname{dim}(M)$, with equality holding only in case $M$ is a flat torus. Finally, Milnor [Mil] and Wolf [Wol] (also see [Gro]) proved that the fundamental group of a compact manifold $M$ with nonnegative Ricci curvature has polynomial growth of degree $d$, where $d \leq \operatorname{dim}(M)$.

In this paper, we wish to refine the upper bound $\operatorname{dim}(M)$ in both inequalities mentioned above for manifolds with nonnegative Ricci curvature and infinite fundamental group. The refinement replaces $\operatorname{dim}(M)$ by $\operatorname{cat}(M)$, the LusternikSchnirelmann category of $M$. The arguments are quite simple and follow directly from the fact that the Cheeger-Gromoll splitting theorem for nonnegative Ricci curvature manifolds gives a splitting $T^{r} \times N$ of a finite cover of $M$, where $N$ is simply connected. The refined bound on $b_{1}(M)$ is then an easy topological consequence of the hard geometry underlying the Cheeger-Gromoll result. In spite of this, we think these refined bounds are interesting and we do not believe they have been noted before. We shall also see that, in the case of symplectic manifolds with nonnegative Ricci curvature, the refined inequalities are actually the best possible. Because category is not a common tool in geometry, we first give a short review of it and then proceed to the results.

Received by the editors June 9, 2000 and, in revised form, August 24, 2000.

1991 Mathematics Subject Classification. Primary 53P99; Secondary 55P99.

I wish to thank Mladen Bestvina for helpful emails pointing out several relevant results in [Gro].

(C)2001 American Mathematical Society 


\section{REVIEW OF CATEGORY}

A space $X$ has $\operatorname{cat}(X)=n$ if and only if $n$ is the least integer such that there is an open covering of $X, U_{1}, \ldots, U_{n+1}$, with each $U_{i}$ contractible to a point in the space $X$. Category was invented by Lusternik and Schnirelmann [LS] as a tool for obtaining a lower bound on the number of critical points of any smooth function on a manifold. In particular, Lusternik and Schnirelmann used category to prove the beautiful fact that the sphere (with any metric) has at least three closed geodesics. Since its invention as a tool in geometric analysis, category has also found a niche in homotopy theory as a bound for various homotopical invariants (see $[\mathrm{Fe}]$ or $[\mathrm{Wh}]$ ).

Properties 2.1. The basic properties of category which we shall use are the following (see [Ja] for instance).

(1) Category is a homotopy type invariant.

(2) The cup length of a space $X$ is the largest integer $k$ such that there exists a product $x_{1} \cdots x_{k} \neq 0$, with $x_{i} \in H^{*}(X ; A)$. Here the coefficient ring $A$ may vary and the cup length may be considered for any coefficients. In this paper, we shall be concerned only with cup length over $\mathbb{Q}$ or $\mathbb{R}$. It is also easy to see that $\operatorname{cup}(X \times Y)=\operatorname{cup}(X)+\operatorname{cup}(Y)$. The fundamental relation between cup length and category is $\operatorname{cup}(X) \leq \operatorname{cat}(X)$.

(3) An upper bound for category is given by $\operatorname{cat}(X) \leq \operatorname{dim}(X)$ (where, for spaces more general than manifolds, $\operatorname{dim}(X)$ denotes the covering dimension of $X$ ). For CW-complexes (and manifolds in particular), this inequality may be refined to $\operatorname{cat}(X) \leq \operatorname{dim}(X) / r$ where $\pi_{1}(X)=\ldots=\pi_{r-1}(X)=0$ (i.e. $X$ is $(r-1)$-connected).

(4) If $\bar{X} \rightarrow X$ is a covering map, then the relation between the categories of $X$ and $\bar{X}$ is $\operatorname{cat}(\bar{X}) \leq \operatorname{cat}(X)$.

(5) There is a general product inequality for category. Namely, $\operatorname{cat}(X \times Y) \leq$ $\operatorname{cat}(X)+\operatorname{cat}(Y)$.

These basic properties of category are all we need from homotopy theory to prove our refined bounds.

\section{The main estimate}

The main tool we use is the following consequence of the Cheeger-Gromoll Splitting Theorem [CG] (also see [Pet] for an exposition).

Theorem 3.1 (Cheeger-Gromoll Splitting). If $M$ is a compact manifold with nonnegative Ricci curvature, then there is a finite cover $\bar{M}$ of $M$ with a Riemannian splitting $\bar{M} \cong T^{r} \times N$. Further, $N$ is simply connected and $T^{r}$ is flat.

Remark 3.2. In the theorem, it could be the case that $r=0$. Then, since $N$ is simply connected and the covering is finite, $\pi_{1}(M)$ would have to be finite. We exclude this case below and focus only on manifolds with infinite fundamental groups. Also note that, because the covering $p: \bar{M} \rightarrow M$ is finite, the induced homomorphism $p^{*}: H^{*}(M ; \mathbb{Q}) \hookrightarrow H^{*}(\bar{M} ; \mathbb{Q})$ is an injection. This is so because a finite covering of degree $m$ has associated to it a transfer homomorphism $\tau: H^{*}(\bar{M} ; \mathbb{Q}) \rightarrow H^{*}(M ; \mathbb{Q})$ with the property that $\tau \circ p^{*}(\alpha)=m \cdot \alpha$ (see $[\mathrm{Ad}]$ for an exposition). For $\mathbb{Q}$ coefficients, multiplication by $m$ is an isomorphism, so $p^{*}$ is a (split) injection. Therefore, in particular, we see that $b_{1}(M) \leq b_{1}(\bar{M})=b_{1}\left(T^{r}\right)=r$. 
The refined estimates for the first Betti number and for the growth degree of the fundamental group are immediate consequences of the Cheeger-Gromoll splitting.

Theorem 3.3 (Main estimate). Suppose $M^{n}$ is a compact manifold with nonnegative Ricci curvature, infinite fundamental group and Cheeger-Gromoll splitting $\bar{M}=T^{r} \times N$. Then

$$
\operatorname{cat}(M) \geq r+\operatorname{cup}(N) \geq b_{1}(M)+\operatorname{cup}(N)
$$

where $b_{1}(M)$ is the first Betti number of $M$.

Proof. We simply apply the basic cup length and covering estimates of category given by Property 2.1 (2) and Property 2.1 (4).

$$
\begin{aligned}
\operatorname{cat}(M) & \geq \operatorname{cat}(\bar{M}) \\
& \geq \operatorname{cup}(\bar{M}) \\
& =\operatorname{cup}\left(T^{r}\right)+\operatorname{cup}(N) \\
& =r+\operatorname{cup}(N) .
\end{aligned}
$$

Of course, we know that $b_{1}(M) \leq b_{1}(\bar{M})=r$ by Remark 3.2 , so this gives the second inequality.

This almost trivial estimate relating cat $(M)$ to $r$ and $b_{1}(M)$ may now be applied to refine two of the most well-known results about nonnegative Ricci curvature manifolds.

Corollary 3.4. For $M$ as in Theorem [3.3;

(1) $b_{1}(M) \leq \operatorname{cat}(M)$. Further, if $b_{1}(M)=\operatorname{cat}(M)$, then $M$ is a flat torus.

(2) The degree of growth of $\pi_{1}(M)$ is bounded above by $\operatorname{cat}(M)$.

Proof. The proof of (1) is immediate except for the case $b_{1}(M)=\operatorname{cat}(M)$. Suppose $b_{1}(M)=\operatorname{cat}(M)$. Then we get

$$
\begin{aligned}
\operatorname{cat}(M) & \geq b_{1}(M)+\operatorname{cup}(N) \\
& \geq \operatorname{cat}(M)+\operatorname{cup}(N) \\
0 & \geq \operatorname{cup}(N) .
\end{aligned}
$$

But this means that $\operatorname{cup}(N)=0$ for rational coefficients and this is impossible for a compact manifold unless it is a point. Hence, $\bar{M}=T^{n}$. This implies that $M=$ $K(\pi, 1)$ and we know that $\operatorname{cat}(M)=\operatorname{dim}(M)$ in that case. Thus, $b_{1}(M)=\operatorname{dim}(M)$ and we are back to the standard 'Bochner' condition for $M$ to be a flat torus (see [LM] or [Pet] for example).

For (2), note that the degree of growth of a free abelian group is its rank, so the degree of growth of $\pi_{1}(\bar{M})=\pi_{1}\left(T^{r}\right)$ is $r$. Also, the degree of growth for a group and a subgroup of finite index are the same (see [Gro]), so the degree of growth of $\pi_{1}(M)$ is also $r$. But Theorem 3.3 then gives $r \leq \operatorname{cat}(M)$.

Remark 3.5. The Bochner technique shows that, for $M$ of nonnegative Ricci curvature, $b_{1}(M)$ is the dimension of the space of parallel vector fields on $M$. While it is obvious why $b_{1}(M)$ should then be bounded above by $\operatorname{dim}(M)$, the upper bound $\operatorname{cat}(M)$ is more mysterious (even in light of Corollary 3.4) and should be investigated more extensively. 


\section{C-SympleCtiC MANifOLdS}

A $2 n$-manifold $M^{2 n}$ is c-symplectic if it has a cohomology class $\omega \in H^{2}(X ; \mathbb{Q})$ such that $\omega^{n} \neq 0$. These manifolds are called c-symplectic because they mimic symplectic manifolds cohomologically (see [LO). If a c-symplectic manifold is simply connected, then its category is easy to determine. By Property 2.1 (2), we have $\operatorname{cat}(M) \geq \operatorname{cup}(M) \geq n$ and, by Property $2.1(3), \operatorname{cat}(M) \leq \operatorname{dim}(M) / 2=n$. Hence, $\operatorname{cat}(M)=n=\operatorname{dim}(M) / 2$. Non-simply connected c-symplectic manifolds of dimension $2 n$ may have any category between $n$ and $2 n$ (see $[\mathrm{RO}$ for a case when $\operatorname{cat}(M)=\operatorname{dim}(M))$. We shall see this in Example 4.6. When $M$ in Theorem 3.3 is c-symplectic, we can obtain a much better bound on $b_{1}(M)$. In order to see how this comes about, we need a few facts about finite coverings of c-symplectic manifolds.

Lemma 4.1. Suppose $p: \bar{M} \rightarrow M^{2 n}$ is a finite covering map and $M$ is c-symplectic with c-symplectic class $\omega$. Then the following properties hold:

(1) $\bar{M}$ is c-symplectic.

(2) If $\bar{M}=T^{r} \times N$ with $N$ simply connected, then $r=2 k$, $\operatorname{dim}(N)=2(n-k)$, $\omega=\omega_{1}+\omega_{2}$ with $\omega_{1} \in H^{2}\left(T^{2 k} ; \mathbb{Q}\right), \omega_{2} \in H^{2}(N ; \mathbb{Q})$ and $\omega_{1}^{k} \neq 0, \omega_{2}^{n-k} \neq 0$.

In particular, for a c-symplectic manifold $M$ with nonnegative Ricci curvature, the Cheeger-Gromoll splitting has the form $\bar{M}=T^{2 k} \times N$ with $N$ simply connected and c-symplectic.

Proof. For $(1)$ we use the fact that $H^{*}(M ; \mathbb{Q})$ injects into $H^{*}(\bar{M} ; \mathbb{Q})$ (see Remark 3.2). Hence, $\omega$ and $\omega^{n}$ lift to the cover nontrivially. Because $\bar{M}$ is a finite cover, $\operatorname{dim}(\bar{M})=2 n=\operatorname{dim}(M)$, so $\omega^{n} \neq 0$ is a top class.

For $(2)$, note that, since $N$ is simply connected, $H^{2}(\bar{M} ; \mathbb{Q})=H^{2}\left(T^{r} ; \mathbb{Q}\right) \oplus$ $H^{2}(N ; \mathbb{Q})$. Thus, $\omega=\omega_{1}+\omega_{2}$. Then $\omega^{n}=\left(\omega_{1}+\omega_{2}\right)^{n} \neq 0$ splits as a sum of terms involving products of powers of $\omega_{1}$ and $\omega_{2}$, at least one term of which is nonzero. Take $\omega_{1}^{s} \omega_{2}^{n-s} \neq 0$. Suppose $2 s<r$. Then $n-s>n-r / 2$ and, consequently, $\left|\omega_{2}^{n-s}\right|>2 n-r$. But $\operatorname{dim}(\bar{M})=\operatorname{dim}\left(T^{r}\right)+\operatorname{dim}(N)$, so $\operatorname{dim}(N)=2 n-r$ and $\omega_{2}^{n-s}$ must vanish, contradicting the nontriviality of $\omega_{1}^{s} \omega_{2}^{n-s}$. On the other hand, if $r<2 s$, then $\omega_{1}^{s}=0$, again contradicting the nontriviality of $\omega_{1}^{s} \omega_{2}^{n-s}$. Therefore, $r=2 s$ and the rest follows.

Remark 4.2. There are two things to note here.

- While all symplectic manifolds are c-symplectic (see [MS]), the notion of c-symplectic is definitely more general than symplectic. For example, while $\mathbb{C P}^{2} \# \mathbb{C P}^{2}$ is c-symplectic, it is certainly not symplectic (see [Au] or [TO]).

- Calabi-Yau manifolds are Kähler manifolds which are Ricci flat (or, equivalently, whose first Chern class vanishes). These manifolds, which therefore are symplectic with nonnegative Ricci curvature, are known to exist in all (complex) dimensions. See [Yau for example. Other symplectic manifolds of nonnegative Ricci curvature may be found in Example 4.6

Theorem 4.3 (Main estimate for c-symplectic manifolds). Suppose $\left(M^{2 n}, \omega\right)$ is a closed c-symplectic manifold with nonnegative Ricci curvature, infinite fundamental group and Cheeger-Gromoll splitting $\bar{M}=T^{2 k} \times N$ (as in Lemma 4.1). Then

$$
2 \operatorname{cat}(M)-\operatorname{dim}(M) \geq 2 k \geq b_{1}(M)
$$

where $b_{1}(M)$ is the first Betti number of $M$. 
Proof. Note that, because $N^{2(n-k)}$ is c-symplectic and simply connected, we have

$$
n-k \leq \operatorname{cup}(N) \leq \operatorname{cat}(N) \leq \frac{1}{2} \operatorname{dim}(N)=n-k
$$

so that $\operatorname{cup}(N)=n-k$. Then

$$
\begin{aligned}
2 \operatorname{cat}(M) & \geq 2 \operatorname{cat}(\bar{M}) \\
& \geq 2 \operatorname{cup}(\bar{M}) \\
& =2 \operatorname{cup}\left(T^{2 k}\right)+2 \operatorname{cup}(N) \\
& =4 k+2(n-k) \\
& =2 k+2 n \\
& =2 k+\operatorname{dim}(M) .
\end{aligned}
$$

Of course, we know that $b_{1}(M) \leq b_{1}(\bar{M})=2 k$, so this gives the second inequality.

So now we have the following refined estimates.

Corollary 4.4. For $M$ as in Theorem 4.3.

(1) The dimension of the space of parallel vector fields on $M$ is less than or equal to $2 \operatorname{cat}(M)-\operatorname{dim}(M)$.

(2) The degree of growth of $\pi_{1}(M)$ is bounded above by $2 \operatorname{cat}(M)-\operatorname{dim}(M)$.

Remark 4.5. Note that $2 \operatorname{cat}(M)-\operatorname{dim}(M)<\operatorname{cat}(M)$ unless $\operatorname{cat}(M)=\operatorname{dim}(M)$. Manifolds with $\operatorname{cat}(M)=\operatorname{dim}(M)$ have very special properties, in some sense akin to those of aspherical manifolds (see [OW]).

The following examples show that the estimates above are best possible for csymplectic manifolds with nonnegative Ricci curvature.

Example 4.6. There are symplectic manifolds $M^{2 n}$ of nonnegative Ricci curvature having category anywhere between the cup length estimate $n$ and the dimension $2 n$. For a simply connected, symplectic manifold $N^{2 n-2 j}$ of nonnegative Ricci curvature (e.g.

$$
\left.N=\mathbb{C P}^{n-j} \quad \text { or } \quad N=\prod_{t=1}^{n-j} S^{2}\right),
$$

define a $2 n$-dimensional symplectic nonnegative Ricci curvature manifold

$$
M_{j}^{2 n}=N^{2 n-2 j} \times T^{2 j}
$$

and note that Property 2.1 (2) and Property 2.1 (5) show

$$
\operatorname{cat}\left(M_{j}\right)=n+j
$$

for $0 \leq j \leq n$. Specifically, we have

$$
\begin{aligned}
\operatorname{cup}\left(N \times T^{2 j}\right) & \leq \operatorname{cat}(M) \leq \operatorname{cat}(N)+\operatorname{cat}\left(T^{2 j}\right), \\
n+j=n-j+2 j & \leq \operatorname{cat}(M) \leq n-j+2 j=n+j .
\end{aligned}
$$

This shows that c-symplectic (or even symplectic manifolds) with nonnegative Ricci curvature realize all category values between $\operatorname{dim}(M) / 2$ and $\operatorname{dim}(M)$. This example also shows that it is not true that $b_{1}(M)=2 \operatorname{cat}(M)-\operatorname{dim}(M)$ implies $M$ is a flat torus. To see this, observe that $b_{1}\left(M_{j}\right)=2 j$ and $2 \operatorname{cat}\left(M_{j}\right)-\operatorname{dim}\left(M_{j}\right)=$ $2(n+j)-2 n=2 j$ also. 
Problem 4.7. Give an example of a c-symplectic manifold $M$ with infinite fundamental group and nonnegative Ricci curvature such that $b_{1}(M)<2 \operatorname{cat}(M)-$ $\operatorname{dim}(M)$.

The Bochner bound $b_{1}(M) \leq \operatorname{dim}(M)$ obstructing the existence of a metric on $M$ with nonnegative Ricci curvature may be overcome by taking products. Specifically, suppose a (compact) manifold $M^{n}$ has $b_{1}(M)>\operatorname{dim}(M)=n$. Choose a (compact) simply connected manifold $N^{s}$ with $b_{1}(M \times N)=b_{1}(M) \leq n+s=\operatorname{dim}(M \times N)$. Then, although $M$ has no metric with nonnegative Ricci curvature, the product $M \times N$ exhibits no Bochner obstruction to having such a metric. The following result shows that this behavior cannot happen in the context of c-symplectic manifolds with the upper bound $b_{1}(M) \leq 2 \operatorname{cat}(M)-\operatorname{dim}(M)$.

Proposition 4.8. Suppose $\left(M^{2 n}, \omega\right)$ is a c-symplectic manifold with $b_{1}(M)>$ $2 \operatorname{cat}(M)-\operatorname{dim}(M)$. Then, for any simply connected c-symplectic manifold $N^{2 s}$,

$$
b_{1}(M \times N)>2 \operatorname{cat}(M \times N)-\operatorname{dim}(M \times N) .
$$

Therefore, for any such $N$, the product $M \times N$ cannot have nonnegative Ricci curvature.

Proof. We know that $\operatorname{cat}(M \times N) \leq \operatorname{cat}(M)+\operatorname{cat}(N)$ and $\operatorname{cat}(N)=s$ by Property 2.1 (5), Property 2.1 (2) and Property 2.1 (3). Thus

$2 \operatorname{cat}(M \times N)-\operatorname{dim}(M \times N) \leq 2 \operatorname{cat}(M)+2 s-2(n+s)=2 \operatorname{cat}(M)-\operatorname{dim}(M)$.

Because $b_{1}(M \times N)=b_{1}(M)$, we obtain

$$
\begin{aligned}
b_{1}(M \times N) & =b_{1}(M) \\
& >2 \operatorname{cat}(M)-\operatorname{dim}(M) \\
& \geq 2 \operatorname{cat}(M \times N)-\operatorname{dim}(M \times N) .
\end{aligned}
$$

Remark 4.9. There have been many papers which give generalizations of the Cheeger-Gromoll splitting to almost nonnegative Ricci curvature manifolds. In particular, in [Cai], it is shown that there are $\epsilon>0, i_{0}>0$ so that, for a manifold $M$, the conditions

$$
\operatorname{Ricci}(M) \geq-\epsilon, \quad \operatorname{diam}(M) \leq 1, \quad \operatorname{Injectivity} \operatorname{radius}(M) \geq i_{0}
$$

provide a (diffeomorphic) splitting of a finite cover $\bar{M}=T^{r} \times N$. The results presented here have obvious generalizations to these situations which the reader can formulate.

Remark 4.10. The $h$-rank of a space $X$ is the rank of $h(G(X))$, where $h: \pi_{1}(X) \rightarrow$ $H_{1}(X ; \mathbb{Z})$ is the Hurewicz homomorphism and $G(X)$ is the Gottlieb group. Recall that $G(X)=$ Image $\left(\mathrm{ev}_{\#}: \pi_{1}\left(X^{X}, 1_{X}\right) \rightarrow \pi_{1}(X)\right)$ where ev: $X^{X} \rightarrow X$ is the evaluation map given by $\operatorname{ev}(f)=f\left(x_{0}\right)$. In [Go], Op (also see [OP]), it was shown that a space with h-rank equal to $r$ has a finite abelian cover $\bar{X} \rightarrow X$ with $\bar{X} \simeq T^{r} \times Y$ for some $Y$. The same arguments as those above (with small modifications such as assuming the simply-connectedness of $Y$ for instance) show the following.

Theorem 4.11. Suppose $M$ is c-symplectic with $h$ - $\operatorname{rank}(M)=r$. Then $r=2 k$ and

$$
\text { h-rank }(M) \leq 2 \operatorname{cat}(M)-\operatorname{dim}(M) .
$$


Of course, h-rank $(M) \leq b_{1}(M)$, but the result does not assume nonnegative Ricci curvature. In some sense then, the condition on the Gottlieb group replaces the curvature requirement.

\section{REFERENCES}

[Ad] J. F. Adams, Infinite Loop Spaces, Annals of Math. Studies 90, Princeton Univ. Press 1978. MR 80d:55001

[Au] M. Audin, Exemples de variétés presque complexes, Enseign. Math. 37 (1991) 175-190. MR 92g:53024

[Cai] M. Cai, A splitting theorem for manifolds of almost nonnegative Ricci curvature, Ann. Global Anal. Geom. 11 no. 4 (1993) 373-385. MR 94j:53043

[CG] J. Cheeger and D. Gromoll, The splitting theorem for manifolds of non-negative Ricci curvature, J. Diff. Geom. 6 (1971) 119-128. MR 46:2597

[DoC] M. Do Carmo, Riemannian Geometry, Birkhäuser Boston, 1992. MR 92i:53001

[Fe] Y. Felix, La Dichotomie Elliptique-Hyperbolique en Homotopie Rationnelle, Asterisque 176, Soc. Math. France, 1989. MR 91c:55016

[GHL] S. Gallot, D. Hulin and J. Lafontaine, Riemannian Geometry, Universitext, SpringerVerlag, 1990. MR 91j:53001

[Go] D. Gottlieb, Splitting off tori and the evaluation subgroup of the fundamental group, Israel J. Math. 66 (1989) 216-222. MR 90j:55019

[Gro] M. Gromov, Metric Structures for Riemannian and non-Riemannian Spaces, Birkhäuser Boston, 1999. MR 2000d:53065

[Ja] I. James, On category in the sense of Lusternik-Schnirelmann, Topology 17 (1978), 331348. MR 80i:55001

[LM] H. B. Lawson and M. L. Michelsohn, Spin Geometry, Princeton Math. Series 38, Princeton Univ. Press, 1989. MR 91g:53001

[LO] G. Lupton, and J. Oprea, Cohomologically symplectic spaces: toral actions and the Gottlieb group, Trans. Amer. Math. Soc. 347 no. 1 (1995), 261-288. MR 95f:57056

[LS] L. Lusternik and L. Schnirelmann, Methodes Topologiques Dans Les Problemes Variationnels, Actualites Scientifiques et Industrielles 188, Hermann, 1934.

[Mil] J. Milnor, A note on curvature and fundamental group, J. Diff. Geom. 2 (1968) 1-7. MR 38:636

[MS] D. McDuff and D. Salamon, Introduction to Symplectic Topology, 2nd ed., Oxford University Press, 1998. MR 2000g:53098

[Op] J. Oprea, A homotopical Conner-Raymond theorem and a question of Gottlieb, Can. Math. Bull. 33 (1990), 219-229. MR 91e:55019

[OP] J. Oprea and J. Pak, Principal bundles over tori and maps which induce the identity on homotopy, Top. Appl. 52 (1993), 11-22. MR 95e:55024

[OW] J. Oprea and J. Walsh, Quotient maps, group actions and Lusternik-Schnirelmann category, preprint 2000, to appear in Top. Appl.

[Pet] P. Petersen, Riemannian Geometry, Grad. Texts in Math., 171, Springer-Verlag, 1998. MR 98m:53001

[RO] Y. Rudyak and J. Oprea, On the Lusternik-Schnirelmann category of symplectic manifolds and the Arnold conjecture, Math. Zeit. 230 no. 4 (1999) 673-678. MR 2000b:53115

[TO] A. Tralle and J. Oprea, Symplectic Manifolds with no Kähler Structure, Lecture Notes in Math., 1661, Springer-Verlag, 1997. MR 98k:53038

[Wh] G. Whitehead, Elements of Homotopy Theory, Grad. Texts in Math., 61, Springer-Verlag, 1978. MR 80b:55001

[Wol] J. Wolf, Growth of finitely generated solvable groups and curvature of Riemannian manifolds, J. Diff. Geom. 2 (1968), 421-426. MR 40:1939

[Yau] S.-T. Yau, Calabi's conjecture and some new results in algebraic geometry, Proc. Natl. Acad. Sci. 74 (1977), 1798-1799. MR 56:9467

Department of Mathematics, Cleveland State University, Cleveland, Ohio 44115

E-mail address: oprea@math.csuohio.edu 